\title{
Vitamin D intakes in UK South Asian and Caucasian postmenopausal women: a novel longitudinal analysis from 2006-2010
}

\author{
A. L. Darling, O. A. Hakim, K. H. Hart and S. A. Lanham-New \\ Nutritional Sciences Division, University of Surrey, Guildford, GU2 7XH, UK
}

\begin{abstract}
Vitamin D has been linked to increased risk of heart disease, cancer, diabetes, osteoporosis and immune disease ${ }^{(1)}$. Data on UK South Asian intakes on vitamin D are scarce due to their under-representation in major datasets such as the National Diet and Nutrition Survey (NDNS) and the Low Income Nutrition Survey (LINC). The principal aim of this study was to investigate the consistency of vitamin D intake in Caucasian (C) and South Asian (SA) women over a 4 year period. Data was taken from SA and C women in the D-FINES dataset (Vitamin D, Food Intake, Nutrition and Exposure to Sunlight in Southern England). A total of $137 \mathrm{C}$ (mean age 61.3 years $+/-4.5$ ) and $33 \mathrm{SA}$ (mean age $58.8 \mathrm{y}+/-5.3$ ) postmenopausal women completed a four-day diet diary (photograph assisted) in Summer 2006. Of these women, $55 \mathrm{C}$ (mean age 62.3 years $+/-4.6$ ) and $11 \mathrm{SA}$ (mean age 60.1 years $+/-3.8$ ) women completed another food diary in Summer 2010 using the same method as in 2006. Data were analysed for both diaries using Win Diets 2005. At recruitment, none of the South Asian women in the 2006 study were taking a vitamin D supplement, and only $25 \%$ of the returning South Asian women in 2010 had taken a vitamin D supplement at any time since the 2006 measurement. For SA, median (IQR) vitamin D intakes in both Summer 2006 and Summer 2010 were very low at 1.39(1.34) and 0.81(3.18) $\mu \mathrm{g} / \mathrm{d}$ respectively (see figure 1). Median (IQR) vitamin D intakes in C were only slightly higher at 2.45 (2.65) in Summer 2006 and 2.39 (2.36) $\mu \mathrm{g} / \mathrm{d})$ in Summer 2010 (see figure 2). Using PASW (18.0), data were log transformed to adjust for normality and paired-samples $t$-tests showed no statistically significant $(P<0.05)$ differences in vitamin $\mathrm{D}$ intakes in either $\mathrm{C}(t=1.02 ; P=0.315)$ or SA $(t=-0.02 ; P=0.986)$ between the two yearly measurements. However, independent $t$-tests showed there was a significant difference in intakes by ethnicity in 2010 only $((F=18.74) ; P<0.001)$, with SA women having a significantly lower intake, but not in $2006((F=0.002) ; P=0.97)$.
\end{abstract}
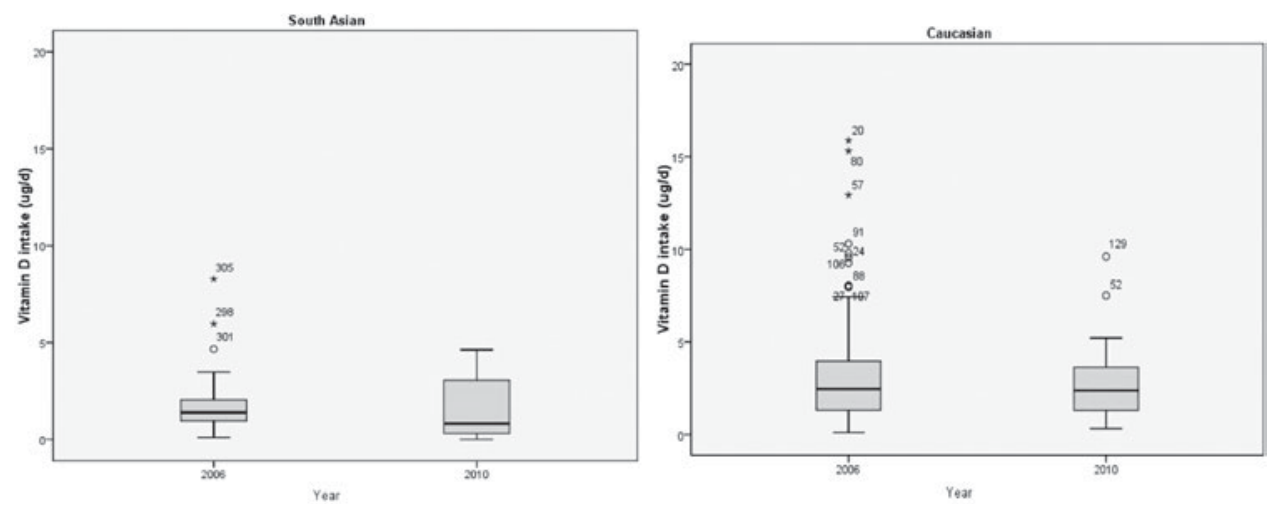

This study emphasises the poor vitamin D intakes in British SA and C women alike and adds novel longitudinal information about the lack of change in vitamin D intake over a period of four years. This is despite increased public, media and clinical attention on the issue of vitamin D in the last few years. The low supplement usage in the SA women is of great concern, considering $10 \mu \mathrm{g} / \mathrm{d}$ is recommended by the $\mathrm{DoH}^{(2)}$ for individuals at high risk of deficiency. Action is required by public health providers to increase awareness of this recommendation in the SA community.

The D-FINES study (2006 data) was funded by the Food Standards Agency (N05064): all views expressed are the authors' own. The 2010 repeat measurement was funded by a University of Surrey Faculty PhD Studentship for AD.

1. Holick MF (2007) N Engl J Med 357(3), 266-81.

2. DOH. Dietary Reference Values for Food Energy and Nutrients for the United Kingdom. Report on Health and Social Subjects No. 41. London: HMSO, 1991. 